\title{
A Criterion for Water Modeling of Non-isothermal Melt Flows in Continuous Casting Tundishes
}

\author{
Chandrashekhar DAMLE and Yogeshwar SAHAI'
}

Formerly Graduate Student, Department of Materials Science and Engineering, The Ohio State University. Now at LTV Steel Research Center, Independence, OH, USA. 1) Department of Materials Science and Engineering, The Ohio State University, Columbus, $\mathrm{OH}$, USA.

(Received on December 7, 1995; accepted in final form on March 4, 1996)

\begin{abstract}
Water modeling of tundish melt flows offers useful insight into the underlying flow phenomena occurring in the tundish. To date most of the water modeling studies have been carried out under isothermal conditions; usually at room temperature. However, fluid flow in continuous casting tundishes is normally not isothermal. By analyzing the dimensionless turbulent Navier-Stokes equation, a modeling criterion is proposed which may be adequate to simulate the flow of molten metal in continuous casting tundishes by means of water modeling. The criterion was tested for a few hypothetical test cases by means of water modeling as well as mathematical modeling. Good agreement between the residence time distribution curves was obtained in the test cases considered when the proposed modeling criterion was satisfied.
\end{abstract}

KEY WORDS: continuous casting; tundish; water modeling; similarity criterion; melt flow; heat transfer.

\section{Introduction}

A knowledge of the fluid flow behavior of liquid steel in a continuous casting tundish is essential for the proper design of tundishes. Direct observation of the flow in a tundish or in a scaled down pilot plant is difficult because of the high temperatures involved and the consequent difficulty in making the observations. Two commonly used methods for characterizing the flow behavior of molten steel in the continuous casting tundish are physical and mathematical modelings. In physical modeling, water is used to simulate the behavior of molten steel. Water modeling is a relatively inexpensive and convenient way to obtain knowledge of the fluid flow behavior of molten steel in a continuous casting tundish. In mathematical modeling, information about the fluid flow patterns is obtained by solving the turbulent Navier-Stokes equation along with the equation of continuity and the equations of the turbulence model being employed. These equations may have to be coupled with the equations for transport of thermal energy and/or mass depending on the flow conditions being modeled. The solution of these equations is obtained numerically. Owing mainly to advances in digital computers, the popularity of mathematical modeling as a means of studying the fluid flow behavior in steelmaking operations has increased significantly in recent years. Several water and mathematical modeling studies have been carried out which have provided deeper insight into the fluid flow phenomena taking place during continuous casting of steel. ${ }^{1-10)}$

Despite advances in mathematical modeling, the im- portance of water modeling should not be discounted. Water modeling experiments are invaluable for flow visualization and in many cases provide the only means of verification of a mathematical model. Several water modeling studies of the fluid flow behavior in continuous casting tundishes have been carried out in the past. ${ }^{1-4)}$ However, practically all of them have ignored the heat transfer aspects and have modeled the flow behavior of steel under isothermal steady state conditions. In actual casting practice, the conditions may not be isothermal. The non-isothermal nature of the flow may be due to heat losses that take place from the top surface and through the walls of the tundish. More importantly, it is also possible that the temperature of the inlet steam into the tundish from the ladle may vary from heat to heat. Thus, there may be situations where the temperature of the inlet stream from the ladle is higher than that of molten steel present in the tundish. These situations are not uncommon in actual casting practice. It has been shown that the fluid flow patterns developed in such a case are quite different from those obtained under isothermal conditions. ${ }^{8-10)}$ Thus, it would be very useful if water modeling could account for the non-isothermal aspects of the fluid flow phenomena taking place in a continuous casting tundish.

To simulate non-isothermal tundish melt flows by means of a water model, thermal similarity must exist between the model and the prototype. To attain thermal similarity, the rate of heat transfer by various mechanisms in the prototype and the model have to bear a constant ratio to each other. In this paper, a criterion is suggested by which it will be possible to adequately simulate the 
non-isothermal fluid flow behavior taking place in a continuous casting tundish by means of a water model. The criterion was tested for hypothetical test cases involving the fluid flow in a continuous casting tundish. The testing of the criterion was carried out by means of mathematical modeling and also by conducting experiments in a water model of a continuous casting tundish.

\section{Theory}

\subsection{Similarity Requirements for Physical Modeling}

In the case of non-isothermal flows, in addition to the geometric and dynamic similarities, it is necessary to satisfy the thermal similarity. For thermal similarity, the heat transfer characteristics of the model and the prototype should be the same, i.e., the rate of heat loss or generation by various mechanisms should be in the same proportion in the model as in the prototype.

\subsection{Analysis of the Dimensionless Turbulent Navier- Stokes Equation}

The dynamics of fluid flow in any continuous casting tundish are governed by the turbulent Navier-Stokes equation. Using the eddy viscosity concept, and the Boussinessq approximation for the buoyancy force, this may be written in the Cartesian tensor notation as follows:

$$
\begin{aligned}
\frac{\partial\left(\rho u_{i}\right)}{\partial t}+\frac{\partial\left(\rho u_{i} u_{j}\right)}{\partial x_{j}}= & \frac{\partial}{\partial x_{j}}\left[\mu_{\text {eff }}\left(\frac{\partial u_{i}}{\partial x_{j}}+\frac{\partial u_{j}}{\partial x_{i}}\right)\right] \\
& -\frac{\partial p}{\partial x_{i}}+\left(\rho-\rho_{\text {ref }}\right) g_{i} \ldots . . .
\end{aligned}
$$

The effective viscosity $\mu_{\text {eff }}$, in the above equation is the sum of the molecular and turbulent viscosities. The last term in the above equation accounts for the buoyancy force per unit volume that arises due to the differences in density throughout the body of the fluid. If $\beta$ is the coefficient of thermal expansion of the fluid,

$$
\beta=\frac{-1}{\rho_{\mathrm{ref}}}\left(\frac{\partial \rho}{\partial T}\right)_{p}
$$

Therefore, for small variations in density,

$$
\rho-\rho_{\text {ref }}=-\rho_{\text {ref }} \beta \Delta T
$$

In the above equations, $\rho_{\text {ref }}$ is some reference density. The Navier-Stokes equations may now be rewritten as

$$
\begin{aligned}
\frac{\partial\left(\rho u_{i}\right)}{\partial t}+\frac{\partial\left(\rho u_{i} u_{j}\right)}{\partial x_{j}}= & \frac{\partial}{\partial x_{j}}\left[\mu_{\text {eff }}\left(\frac{\partial u_{i}}{\partial x_{j}}+\frac{\partial u_{j}}{\partial x_{i}}\right)\right] \\
& -\frac{\partial p}{\partial x_{i}}-\rho_{\text {ref }} \beta \Delta T g_{i} \ldots \ldots .
\end{aligned}
$$

The temperature distribution within a turbulent flowing system is determined by the equation for the conservation of thermal energy.

$$
\frac{\partial \rho C_{p} T}{\partial t}+\frac{\partial\left(\rho C_{p} T u_{j}\right)}{\partial x_{j}}=\frac{\partial}{\partial x_{j}}\left(k_{\text {eff }} \frac{\partial T}{\partial x_{j}}\right)
$$

In the above equation, $k_{\text {eff }}$ is the effective thermal conductivity, which is the sum of the molecular and turbulent thermal conductivities of the fluid. The temperature field is coupled with the velocity field through the buoyancy force term in Eq. (4).

Equations (4) and (5) together govern the dynamics of the flow within the system. In addition to the above equations, the boundary conditions also have a strong influence on the flow and temperature field within the tundish. For the momentum equation, the no-slip boundary condition is imposed along the tundish walls. The top slag layer is assumed to have a negligible effect on the flow field and therefore, a free surface boundary condition is imposed along the top surface.

For the temperature equation, two types of boundary conditions are permissible - either the boundary temperature, or the boundary heat flux may be fixed along any portion of the boundary. ${ }^{11)}$ For tundish flows, heat losses take place through the top surface and through the bottom and side walls. Heat is input into the system through the inlet. The temperature of the inlet stream may be nearly constant for pouring from ladles with insulated tops. ${ }^{12)}$ Therefore, the boundary condition at the inlet is

$$
T=T_{\text {inlet }}
$$

Along the other parts of the boundary, a constant heat flux may be assumed. However, in many modern tundish operations, the tundish is covered. Under these conditions, the heat losses from the tundish may be neglected. Therefore in this work, heat losses through the top slag layer and through the tundish walls were not considered.

In order to render the above equations in the dimensionless form, it is necessary to define appropriate scales for the different variables. If $U$ is taken to be some reference velocity, $L$ to be a reference length, and $T_{0}$ the initial temperature for the system being studied, then the following non-dimensional quantities may be defined:

$$
\begin{aligned}
& \text { velocity, } u^{*}=\frac{u}{U} \\
& \text { length, } x_{i}^{*}=\frac{x_{i}}{L} \\
& \text { density, } \quad \rho^{*}=\frac{\rho}{\rho_{\text {ref }}} \\
& \text { pressure, } \quad p^{*}=\frac{p-p_{\mathrm{ref}}}{\rho_{\mathrm{ref}} U^{2}} \\
& \text { time, } t^{*}=\frac{t U}{L} \\
& \text { temperature, } \quad T^{*}=\frac{T-T_{0}}{T_{\text {inlet }}-T_{0}}=\frac{T-T_{0}}{\Delta T_{0}}
\end{aligned}
$$

The Navier-Stokes equation may now be written using these non-dimensional quantities.

$$
\begin{aligned}
\frac{\partial\left(\rho^{*} u_{i}^{*}\right)}{\partial t^{*}}+\frac{\partial\left(\rho^{*} u_{i}^{*} u_{j}^{*}\right)}{\partial x_{j}^{*}}= & \frac{\partial}{\partial x_{j}^{*}}\left[\frac{\mu_{\mathrm{eff}}}{\rho_{\mathrm{ref}} U L}\left(\frac{\partial u_{i}^{*}}{\partial x_{j}^{*}}+\frac{\partial u_{j}^{*}}{\partial x_{i}^{*}}\right)\right] \\
& -\frac{\partial p^{*}}{\partial x_{i}^{*}}-\frac{\beta \Delta T L}{U^{2}} g_{i} \ldots \ldots \ldots(13)
\end{aligned}
$$


The non-dimensional form of the equation of thermal energy conservation may be written as

$$
\frac{\partial \rho^{*} T^{*}}{\partial t^{*}}+\frac{\partial\left(\rho^{*} T^{*} u_{i}^{*}\right)}{\partial x_{i}^{*}}=\frac{\partial}{\partial x_{i}}\left[\frac{k_{\mathrm{eff}}}{\rho_{\mathrm{ref}} C_{p} U L} \frac{\partial T^{*}}{\partial x_{i}}\right] \ldots
$$

Similarly, the dimensionless inlet boundary condition for heat transfer may be written as

$$
T^{*}=1
$$

The fluid flow in two different systems can become similar only if the solution of the governing equations written in the dimensionless form is identical for both systems. For systems that are geometrically similar, this implies that the dimensionless groups that are present in the dimensionless form of the conservation equations and the boundary conditions should be the same in both systems. The dimensionless groups that arise from the above non-dimensional equations are

$$
\begin{aligned}
& \frac{1}{R e_{\mathrm{t}}}=\frac{\mu_{\mathrm{eff}}}{\rho_{\mathrm{ref}} U L} \ldots \ldots . . . \\
& T u=\frac{\beta \Delta T_{0} L g_{i}}{U^{2}} \ldots \ldots . . \\
& \frac{1}{P r_{\mathrm{t}} \operatorname{Re}_{\mathrm{t}}}=\frac{k_{\text {eff }}}{\rho_{\text {ref }} C_{p} U L}
\end{aligned}
$$

The first of these is very similar to the Reynolds number $(R e)$ in laminar flow, except that the molecular viscosity is replaced by the effective viscosity. Therefore, this number may be taken to be a turbulent Reynolds number, $R e_{t}$. The Reynolds number represents the ratio of the transfer of momentum by inertial and "diffusive" forces. In tundish flows, the inertial force arises due to the momentum of the inlet stream. Since the flow in a tundish is turbulent, the diffusive transfer of momentum takes place through the interaction of turbulent eddies. It should also be noted that under turbulent flow conditions, the Schmidt number, $S c$, is very close to unity. Therefore, $R e_{t}$ is also equal to the ratio of the mass transfer by turbulent diffusion to the mass transfer by convection. Recently, Sahai and Burval, ${ }^{13}$ ) performed tracer dispersion experiments on geometrically similar water models of tundishes. The study was conducted under isothermal conditions. Under such conditions, the only dimensionless group that arises is $R e_{t}$. It was found that the normalized residence time distribution for a pulse input of a tracer was the same under various flow conditions. This is possible only if the ratio of the transfer of the tracer by turbulent diffusion to that by convection is the same under the different conditions. Since the $S c$ is close to unity, this ratio is given by the dimensionless group Eq. (16). Therefore, under isothermal turbulent flow conditions, $R e_{t}$ is constant for geometrically similar systems. Similar results were obtained by Singh and Koria. ${ }^{14)}$ This result is not surprising since it has been found that flow profiles and details of turbulent motion are independent of the molecular Reynolds number (i.e., $R e$ calculated using the molecular viscosity). ${ }^{15,16)}$ Therefore, it stands to reason that $R_{\mathrm{t}}$ should remain constant even under non-isothermal conditions.
The dimensionless number given by Eq. (18) is the product of the turbulent Reynolds and Prandtl numbers. Under turbulent flow conditions, the Prandtl number can be assumed to be constant. ${ }^{17)}$ Also, as discussed above, $R e_{\mathrm{t}}$ is also constant. Therefore, under turbulent flow conditions, the dimensionless group given by Eq. (18) remains constant. Since this is the only dimensionless group that arises from the thermal energy equation and the thermal boundary conditions, under the flow conditions being considered, thermal similarity is automatically satisfied.

The dimensionless group given by Eq. (17) is therefore the only dimensionless group that remains to be satisfied. This quantity is very similar to the modified Froude number. Since this work is pertinent to modeling of tundish flows, and all the quantities in the definition of the dimensionless group pertain to conditions in the tundish, it was decided to call this number as the Tundish Richardson number $(T u)$.

$T u$ denotes the ratio of the buoyancy forces to the inertial forces.

$$
T u=\frac{G r}{R e^{2}}=\frac{g L \beta \Delta T_{0}}{U^{2}}=\frac{\text { buoyancy force }}{\text { inertial force }}
$$

The buoyancy forces arise due to the non-uniform temperature distribution within the tundish. It is these forces that cause the flow profiles within the tundish under non-isothermal conditions to be different from those under isothermal conditions.

Keeping $T u$ constant between the model and the prototype should ensure that the ratio between buoyancy and viscous forces in the two systems is the same. Also, it has been shown earlier that under turbulent flow conditions, no special effort has to be taken to keep the ratio between the inertial and viscous forces the same in the model as in the prototype. Therefore, by keeping $T u$ constant it should be possible to satisfy the requirement of dynamic similarity between the model and the prototype.

\section{Experimental}

From Eq. (17) it can be seen that the important parameters in $T u$ are the characteristic velocity, $U$, the characteristic length, $L$, the temperature difference, $\Delta T_{0}$, and the coefficient of thermal expansion of the fluid, $\beta$. Of these, $\beta$ is a fluid property. The remaining three parameters can be controlled experimentally. The characteristic velocity may be taken as the velocity of the incoming fluid. The characteristic length is the wetted perimeter of the tundish. The temperature difference $\Delta T_{0}$ was taken to be the difference between the temperature of the incoming fluid and the fluid that is initially present in the tundish. To test the validity of the above criterion, several test cases were considered. The values of the control parameters, $U, L$, and $\Delta T_{0}$, used in each of the above test cases along with the boundary conditions used in the mathematical model are listed in Table 1. A schematic representation of the experimental set up used is shown in Fig. 1. The dimensions of the experimental tundish are given in Table 2. 
Table 1. Values of various parameters in $T u$ for the different cases considered.

\begin{tabular}{|c|c|c|c|c|c|c|}
\hline Case & $\begin{array}{l}\text { Tundish } \\
\text { size }\end{array}$ & Fluid & $\begin{array}{c}U \\
\left(\mathrm{~m} \mathrm{~s}^{-1}\right)\end{array}$ & $\begin{array}{c}L \\
(\mathrm{~m})\end{array}$ & $\begin{array}{l}\text { Initial } \\
\text { tempera- } \\
\text { ture } \\
(\mathrm{K})\end{array}$ & $\begin{array}{c}\text { Inlet } \\
\text { stream } \\
\text { tempera- } \\
\text { ture } \\
(\mathrm{K})\end{array}$ \\
\hline 1 & Large & Steel & 1.5 & 1.446 & 1873 & 1898 \\
\hline 2 & Small & Water & 1.1205 & 0.482 & 293 & 311 \\
\hline 3 & Small & Water & 0.747 & 0.482 & 293 & $30 \mathrm{I}$ \\
\hline 4 & Large & Water & 1.92 & 1.446 & 293 & 311 \\
\hline 5 & Large & Water & 1.1205 & 1.446 & 293 & 299 \\
\hline
\end{tabular}

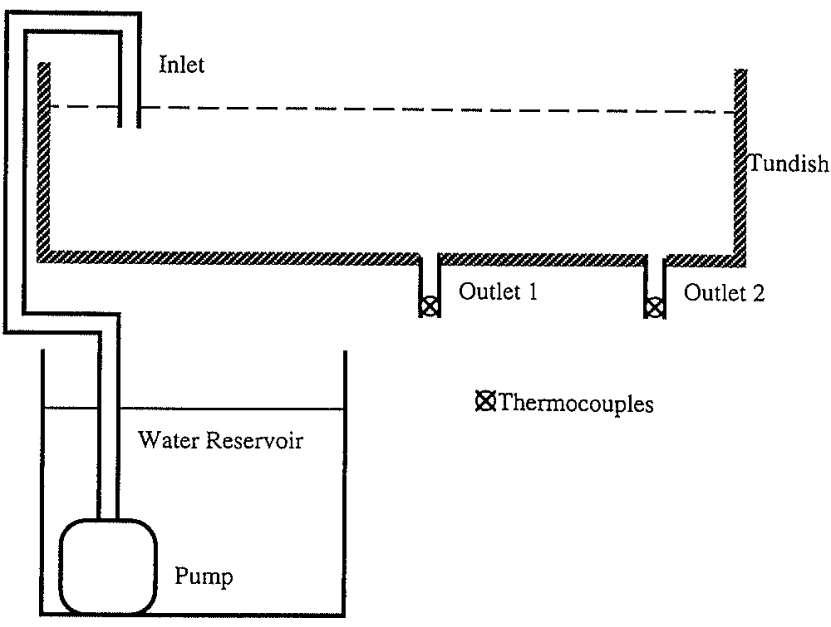

Fig. 1. Schematic diagram of set up used for the water modeling experiments.

Table 2. Dimensions of the various tundishes considered.

\begin{tabular}{lcl}
\hline & Small tundish & Large tundish \\
\hline Length $(\mathrm{m})$ & 1.36 & 4.08 \\
Width $(\mathrm{m})$ & 0.345 & 1.035 \\
Height of fluid $(\mathrm{m})$ & 0.40 & 1.2 \\
Distance between inlet and first & 0.57 & 1.71 \\
$\quad \begin{array}{l}\text { outlet }(\mathrm{m}) \\
\text { Distance between inlet and } \\
\text { second outlet }(\mathrm{m})\end{array}$ & 1.15 & 3.45 \\
\hline
\end{tabular}

Residence time distribution (RTD) curves provide a means of analyzing the overall fluid flow behavior taking place in any reactor. RTD curves are obtained by subjecting the system to a perturbation and then monitoring the response of the system to the perturbation. The perturbation may be either a pulse type or a step type. As mentioned before, the nature of the system being studied in the present work corresponds to a step type of perturbation. Therefore, F-curves ${ }^{18)}$ have been used for analyzing the fluid flow behavior in system being modeled.

In the situation being considered in this work, the temperature of the incoming fluid is greater than that of the fluid initially in the tundish. The RTD curves can then be obtained by monitoring the temperature of the fluid in the exit stream. Under turbulent flow conditions, the Prandtl number and the Schmidt number are very close to unity and thus the dimensionless concentration and temperature distribution should be very similar. Therefore, it was decided to obtain the RTD curves by measuring the temperature of the outlet stream.

The temperature at the outlets was measured using $\mathrm{K}$-type thermocouples. The temperature measured was then converted to the dimensionless form in the following manner:

$$
T_{\text {out }}^{*}=\frac{T_{\text {out }}-T_{0}}{T_{\text {inlet }}-T_{0}}
$$

Dimensionless time was calculated using the following relationship:

$$
t_{0}=\frac{t}{t_{\mathrm{r}}}
$$

where $t_{\mathrm{r}}$ is the mean residence time.

The dimensionless temperature was plotted against dimensionless time to obtain the residence time distribution (RTD) of the fluid in the tundish.

\subsection{Model Formulation}

A mathematical description of the turbulent, nonisothermal, unsteady state fluid flow phenomena is given by the following equations:

(a) Equation of Continuity

(b) Turbulent Navier-Stokes equation

(c) Equation for conservation of thermal energy

(d) The two equations for the $k-\varepsilon$ turbulence model ${ }^{19)}$ Details of these equations can be obtained from Ref. 10).

The density of water as a function of temperature is given by

$$
\rho_{\text {water }}=654.619+2.5446 T-0.004683 T^{2}
$$

where, the temperature $T$ is in Kelvin. The above equation is valid in the temperature range $0-50^{\circ} \mathrm{C}$. It was arrived at on the basis of data obtained from Ref. 20). The density of liquid steel as a function of temperature was calculated using the following relationship:

$$
\rho_{\text {steel }}=7010.0-0.833(T-1808.0)
$$

Again, the temperature $T$ is in Kelvin. Data for the above equation was obtained from Ref. 21). The value of $\beta$ was calculated from the above equations for the two liquids using Eq. (2). For water, an average value of $\beta$ was calculated for the temperature range 20 to $50^{\circ} \mathrm{C}$. This value was then used in all the calculations. The calculated value of $\beta$ for steel was $1.27 \times 10^{-4} \mathrm{~K}^{-1}$, and that of water was $2.95 \times 10^{-4} \mathrm{~K}^{-1}$.

\subsection{Boundary Conditions}

Figure 2 shows the calculation domain used for modeling the fluid flow in the lab-scale tundish (cases 2 and 3 ). The dimensions correspond to those of the tundish used for the water modeling experiments. The full-scale tundish was obtained by scaling up each dimension in the lab-scale tundish by a factor of three. Table 2 gives the dimensions of the tundishes considered in the present work.

For purposes of modeling, only half the tundish was considered due to the symmetry of the flow conditions. A zero normal flux boundary condition was applied for 
all velocity components normal to a boundary. A zero shear stress boundary condition was used in the case of the symmetry boundary and the free surface. The free surface was considered to be flat. A no slip boundary condition was imposed on the velocity components parallel to the walls. The wall shear stress was calculated using the "logarithmic law of the wall."19) No outlet boundary conditions were explicitly specified. The outlet velocity was calculated by the program so as to satisfy an overall mass balance for the system. The temperature and velocity of the incoming stream was specified as per Table 1. The inlet velocity and temperature were kept constant throughout the time period for which the simulations were carried out.

While modeling the flow of water in the tundish, it was assumed that no heat losses took place through any of the surfaces or walls. This assumption is not strictly valid. However, in the temperature range considered the surface heat losses are not significant and therefore, this assumption should not affect the results in any marked way. This was borne out when the results obtained from the mathematical model was compared with the experimental results (to be discussed later).

While modeling the flow of molten steel, again, the heat losses taking place through the various surfaces were neglected, i.e., the surfaces were assumed to be adiabatic.

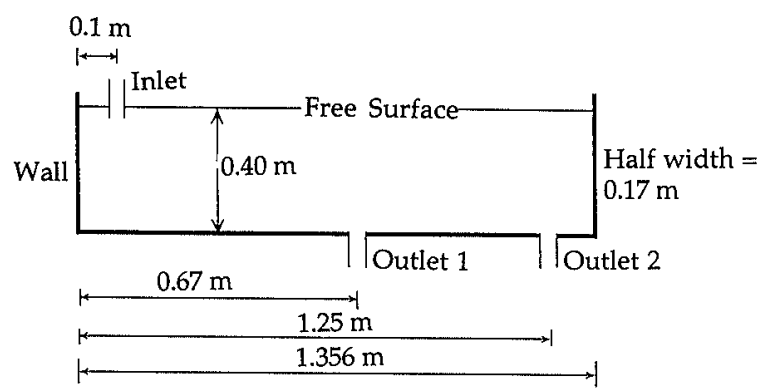

Fig. 2. Calculation domain used for the mathematical modeling simulations.
The inlet temperature was fixed at $1898 \mathrm{~K}$. In each case the initial condition was assumed to be that corresponding to the isothermal steady state situation.

The governing equations along with the boundary conditions were discretized using the control volume procedure. The SIMPLE algorithm was used to resolve the pressure velocity coupling. ${ }^{22)}$ A FORTRAN code was developed by the authors to obtain numerical solutions of the above coupled fluid flow and heat transfer problem. The simulations were carried out on a VAX 8550 computer and also on the CRAY YMP supercomputer located at the Ohio Supercomputer Center. A time step of a tenth of a second was used for the first second after the beginning of pouring from the new ladle. A time step of one second was used for the next three seconds. For the remaining period of the simulation, a time step of four seconds was used.

\section{Results and Discussion}

\subsection{Model Validation}

To test the validity of the mathematical model, the simulations were run for the conditions under which the water modeling experiments were carried out (case 2 and case 3). Figures 3-6 show the experimental as well as the predicted RTD curves obtained at each outlet for the two cases. The agreement between the experimental and the predicted RTD curve is reasonably good. Therefore, the mathematical model was assumed to predict the flow profiles within the tundish to a reasonable degree of accuracy. Thus having established the validity of the mathematical model, the next step was to test the correctness of the modeling criterion by adjusting the values of the different variables in $T u$, but maintaining $T u$ constant.

\subsection{Testing of $T u$ Criterion}

The proposed $T u$ criterion was tested by means of mathematical modeling as well as water modeling.

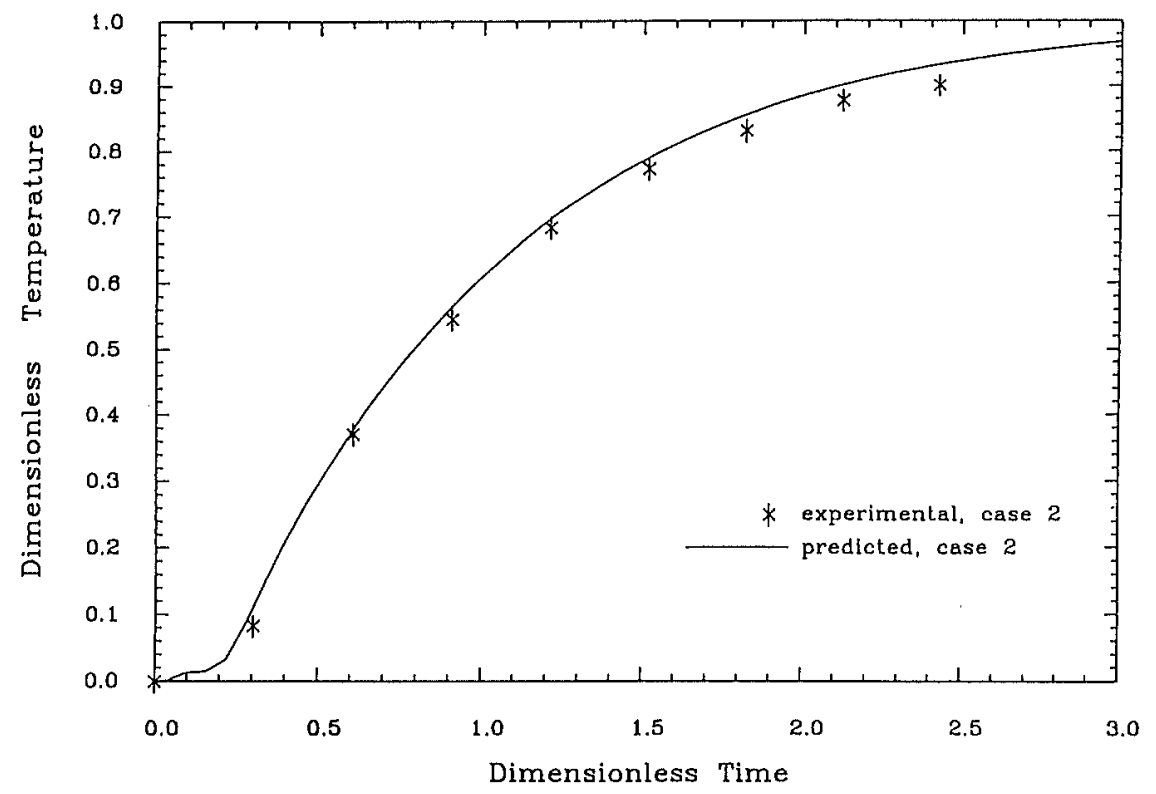

Fig. 3. Comparison between predicted and experimental RTD curve at the near outlet (case 2, Table 1). 


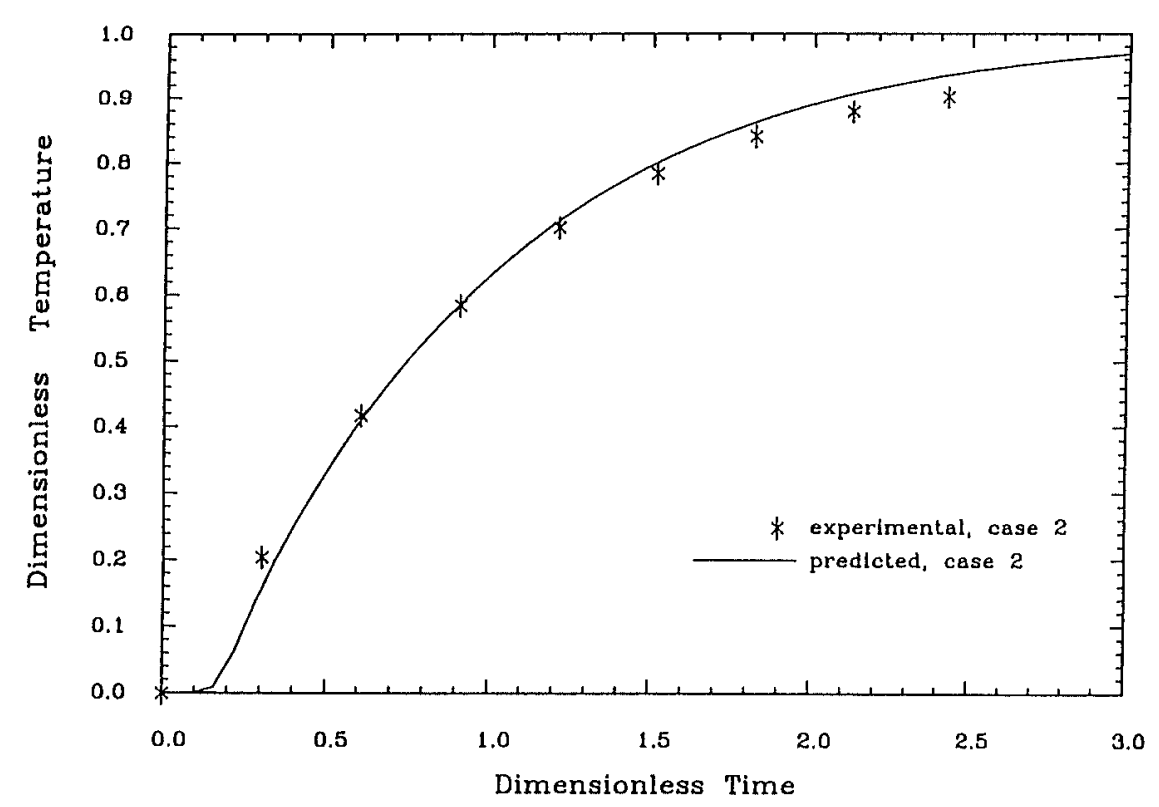

Fig. 4. Comparison between predicted and experimental RTD curve at the far outlet (case 2, Table 1).

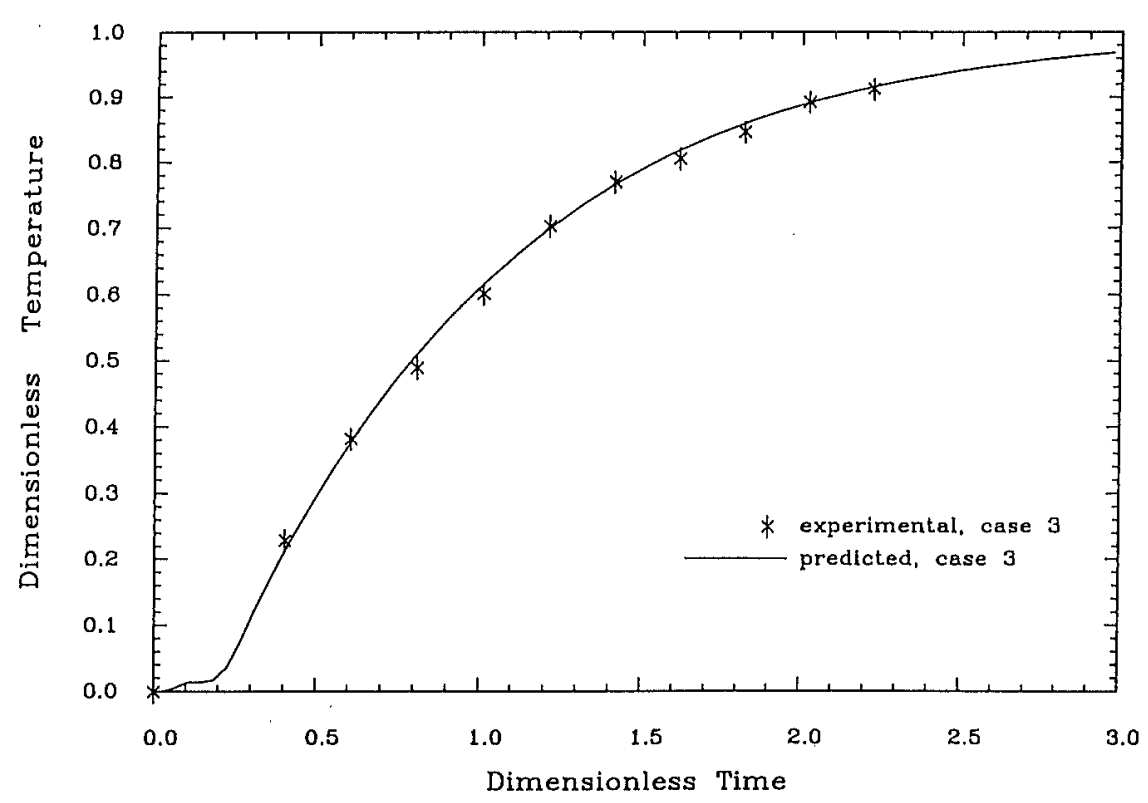

Fig. 5. Comparison between predicted and experimental RTD curve at the near outlet (case 3, Table 1).

Mathematical modeling was first used to simulate the flow of molten steel under typical steady state casting conditions. The values of the various parameters corresponding to this situation are denoted as case 1 in Table 1 . The value of $T u$ for this case was 0.02 . This was used as the standard situation. To test the criterion, the different parameters in $T u$ were then modified ensuring that $T u$ remained constant. RTD curves were obtained by means of mathematical modeling in each case. The different situations considered are given in Table 1. It is seen that the different cases correspond to a wide range of parameters. However, in each case the value of $T u$ is 0.02 . Water modeling was carried out only for cases 2 and 3 . The dimensions of the tundish used for water modeling are shown in Table 2.

Figures 7 and $\mathbf{8}$ show the predicted RTD curves at each of the two outlets of the tundish for the different cases considered in this study. It can be seen from these figures that the RTD curves are practically identical for all the cases. Since the RTD curves reflect the overall fluid flow profiles in the tundish, it can be inferred from the above that the overall flow profiles are similar in each case. This is possible only if all the situations are dynamically similar to each other. Therefore, by keeping $T u$ constant, it may be possible to obtain dynamic similarity between a water model and a prototype steel tundish.

The proposed criterion was also verified by means of water modeling experiments. The experiments were performed under conditions given in case 2 and case 3 in Table 1. Case 2 corresponds to an inlet flow rate of $0.562 \mathrm{ls}^{-1}$ and case 3 corresponds to an inlet flow 
ISIJ International, Vol. 36 (1996), No. 6

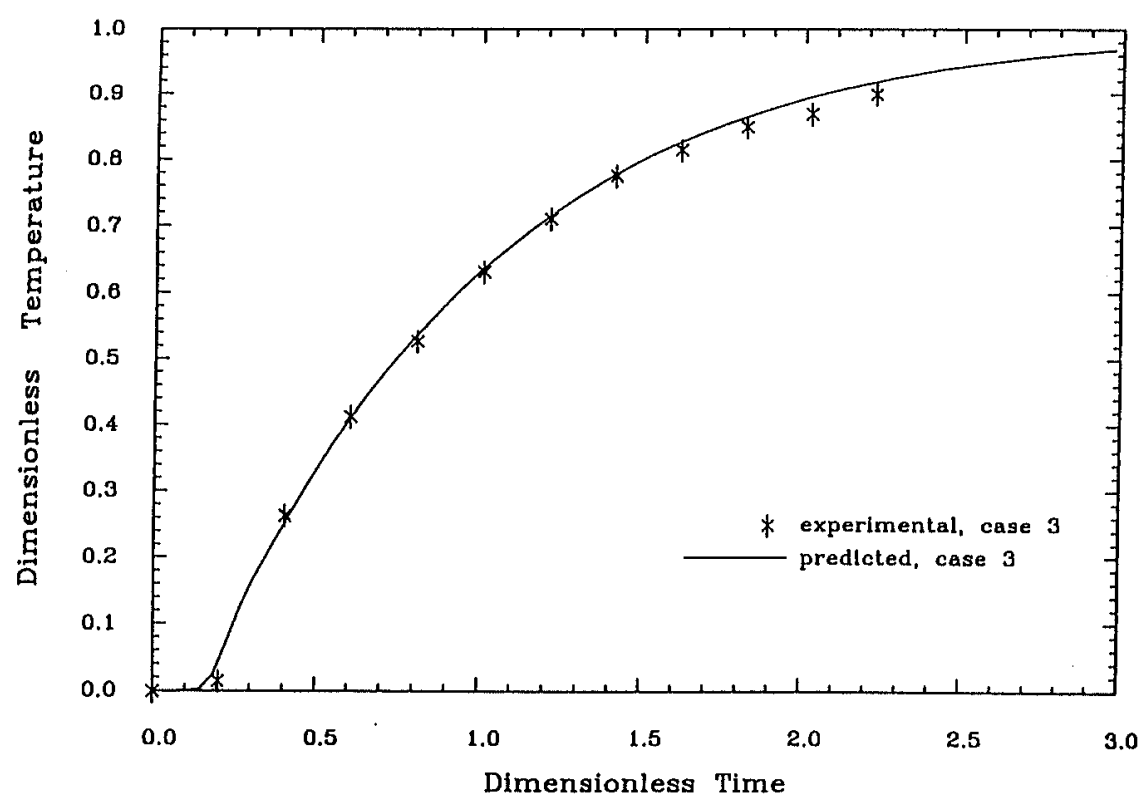

Fig. 6. Comparison between predicted and experimental RTD curve at the far outlet (case 3 , Table 1).

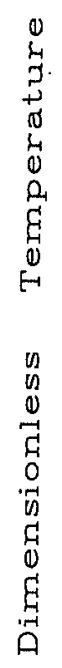


Fig. 7.

Predicted RTD curves for different operating conditions but same value of $T u$ at near outlet.
Fig. 8.

Predicted RTD curves for different operating conditions but same value of $T u$ at far outlet. 


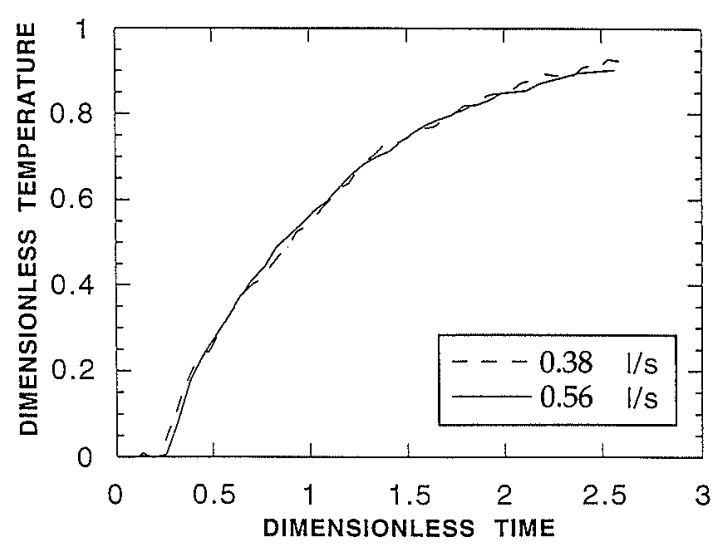

Fig. 9. Experimental RTD curves obtained for two different operating conditions but same $T u$ (case 2 and case 3 , Table 1) at near outlet.

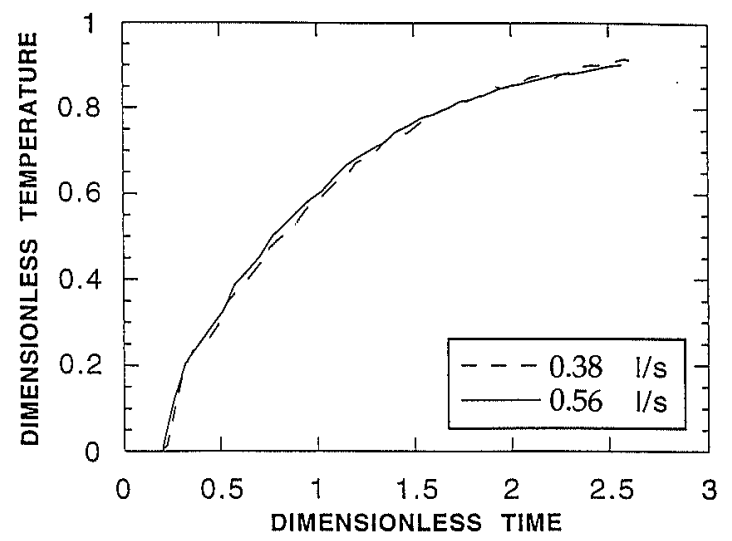

Fig. 10. Experimental RTD curves obtained for two different operating conditions but same $T u$ (case 2 and case 3 , Table 1) at far outlet.

rate of $0.375 / \mathrm{s}^{-1}$. The RTD curves obtained in the two cases are shown in Figs. 9 and 10. Again, it is seen that the two RTD curves are nearly identical. Thus it is seen that under experimental conditions, adherence to the proposed modeling criterion results in almost identical RTD curves for the cases considered. It may thus be concluded that the overall dynamics of the flow in the two cases are the same.

Thus it can be seen that the RTD curves for flow regimes in which $T u$ is the same are identical. Therefore, the overall flow pattern in these different flow regimes may be considered to be the same. In other words, each of these systems is dynamically similar to the other. Therefore, it should be possible to construct a water model to simulate the non-isothermal flow that takes place in continuous casting tundishes that will provide sufficiently accurate information about the nature of the fluid flow phenomena occurring in an actual tundish.

\section{Conclusions}

A criterion has been proposed whereby it should be possible to obtain dynamic similarity between the non-isothermal fluid flow in a prototype and its physical model. The method involves maintaining a dimensionless number, called the 'Tundish Richardson number', $T u$, constant between the prototype and the model. This criterion was arrived at by a dimensional analysis of the turbulent Navier-Stokes equation. Adherence to this criterion should ensure that the ratio of the buoyancy force to the inertial force is constant between the model and the prototype. The proposed criterion has been tested by comparing the RTD curves obtained under different flow conditions for which the value of $T u$ is the same. The testing was carried out by means of a mathematical model as well as by conducting experiments on a water model of a tundish. It has been shown that under these conditions, the overall fluid flow behavior, as reflected by the RTD curves, is the same for the various flow conditions, and therefore, the systems are dynamically similar.

\section{Nomenclature}

$g:$ Acceleration due to gravity $\left(\mathrm{m} \mathrm{s}^{-2}\right)$

$G r$ : Grashof number

$k$ : Thermal conductivity $(\mathrm{W} / \mathrm{m} \cdot \mathrm{K})$

$L$ : Characteristic length dimension $(\mathrm{m})$

$p:$ Pressure $(\mathrm{Pa})$

Pr: Prandtl number

$Q:$ Volumetric flow rate $\left(\mathrm{m}^{3} \mathrm{~s}^{-1}\right)$

Re: Reynolds number

Sc: Schmidt number

$T$ : Temperature (K)

$T_{\text {out }}^{*}$ : Dimensionless temperature in RTD curves

$T_{0}$ : Initial temperature of fluid in Tundish (K)

$T_{\text {inlet }}:$ Temperature of incoming fluid (K)

$T_{\text {out }}:$ Temperature at outlet $(\mathrm{K})$

$t:$ time (s)

$t_{\mathrm{r}}:$ Mean residence time (s)

$t_{0}:$ Dimensionless time in RTD curves

$T u$ : Tundish Richardson number

$u$ : Velocity $\left(\mathrm{m} \mathrm{s}^{-1}\right)$

$U:$ Chracteristic velocity $\left(\mathrm{m} \mathrm{s}^{-1}\right)$

$x$ : Distance coordinate $(\mathrm{m})$

$\beta$ : Coefficient of volume expansion of fluid $\left(\mathrm{K}^{-1}\right)$

$\rho:$ density $\left(\mathrm{kg} \mathrm{m}^{-3}\right)$

$\mu: \quad$ viscosity $\left(\mathrm{kg} \mathrm{m}^{-1} \mathrm{~s}^{-1}\right)$

Subscripts and Superscripts

eff: Sum of molecular and turbulent contributions

ref: Reference quantity

*: Dimensionless quantity

\section{REFERENCES}

1) F. Kemeny, D. J. Harris, A. McLean, J. R. Meadowcraft and J. D. Young: Proc. 2nd Process Technology Conference, ISS-AIME, (1981), 232

2) L. J. Heaslip and A. McLean: Continuous Casting, Vol. 1, ISS-AIME, (1983), 93.

3) J. Knoepke and J. Mastervich: Steelmaking Conf. Proc., Vol. 65, (1982), 3.

4) Y. Sahai and R. Ahuja: Steelmaking Conference Proc., Vol. 69, (1986), 677.

5) K. H. Tacke and J. C. Ludwig: Steel Res., 58 (1987), No. 6, 262.

6) K. Y. M. Lai, M. Salcudean, S. Tanaka and R. I. L. Guthrie: Metall. Trans. B, 17B (1986), No. 9, 449.

7) Y. He and Y. Sahai: Metall. Trans. B, 18B (1987), 81.

8) S. Chakraborty and Y. Sahai: Ironmaking Steelmaking, 19 (1992), No. 6, 479.

9) S. Chakraborty and Y. Sahai: Ironmaking Steelmaking, 19 (1992), No. 6, 488. 
10) S. Chakraborty and Y. Sahai: Metall. Trans. B, 23B (1992), No. 2, (April 1992), 153.

11) J. Szekely, J. K. Brimacombe and J. W. Evans: Mathematical and Physical Modeling of Primary Metals Processing Operations, Wiley, New York, (1988).

12) S. Chakraborty and Y. Sahai: Metall. Trans. B, 23B, No. 2, (April 1992), 135.

13) Y. Sahai and M. Burval: Electric Furnace Conf. Proc., Atlanta, GA, ISS-AIME, (1992), 469.

14) S. Singh and S. C. Koria: Ironmaking Steelmaking, 20 (1993), No. 3, 211.

15) P. Bradshaw: Experiments in Fluids, Vol. 16, (1994), 203.

16) I. Nezu and H. Nakagawa: Turbulence in Open Channel Flows, IAHR-AIRH Monograph series, A. A. Balkema, Rotterdam,
(1993).

17) P. Bradshaw: Turbulence, Topics in Applied Physics, Vol. 12, Springer-Verlag, (1978).

18) O. Levenspiel: Chemical Reaction Engineering, John Wiley \& Sons, Inc., (1972).

19) B. E. Launder and D. B. Spalding: Computer Methods Appl. Mech. Eng., 3 (1974), 269.

20) CRC Handbook of Chemistry and Physics, 70th Ed., R. C. Weast, editor in chief, CRC Press Inc., Boca Raton, Florida, (1990).

21) Smithell's Metals Reference Book, 6th Ed., ed. by E. A. Brandes, Butterworths and Co., (1983).

22) S. V. Patankar: Numerical Heat Transfer and Fluid Flow, Hemisphere Pub. Corp., New York, NY, (1980). 